\title{
Zinc( II) Immune Virucidal Activities for 2019-nCoV Prevention and COVID-19 Respiratory Ailment and Pneumonia
}

\author{
Dr. Sci. Tsuneo Ishida \\ 2-3-6, Saido, Midori-Ku, Saitama-Shi, Saitama-Ken, ̄̄336-0907, Japan \\ *Corresponding Author: Dr. Sci. Tsuneo Ishida, 2-3-6, Saido, Midori-Ku, Saitama-Shi, Saitama-Ken, \\ T336-0907, Japan
}

\begin{abstract}
The zinc-homeostatic immune concentration may provide a protective role against the COVID-19 pandemic, likely by improving the host's resistance against viral infection. 2019-nCoV RNA virus structure at least contains four viral proteins, the spike $(S)$ protein, the membrane $(M)$ protein, the envelope $(E)$ protein, and nucleocapsid $(N)$ protein. When $S 1$ protein recognizes its receptor on human cells, the heptad repeat 1 (HR1) and heptad repeat 2 (HR2) domains are exposed to interact with each other, forming six-helical bundle (6-HB) to mediate membrane fusion between virus and target cell. Factor of present outbreak of disease 19 COVID-19 may be considered to be due to RNA virus mutation that the RNA viruses have high mutation rate and these high rates are correlated with enhanced virulence and evolvability, traits considered beneficial for viruses. Zinc ions could inhibit virus entry and membrane fusion of S1 and S2 domains of spike protein with zinc ion-binding interaction. $\mathrm{Zn}^{2+}$ ions can prevent in the early stage of 2019$n C o V$ infected patient with antiviral zinc homeostatic immunity and have important roles for respirarory and pulmonary process of COVID-19 disease. $\mathrm{Zn}^{2+}$ inhibits coronavirus and anterivirus RNA polymelase activity, and zinc ionophores block the virus replication that the combination of $\mathrm{Zn}^{2+}$ and pyrithione at low concentrations inhibits the replication of SARS-CoV and arterivirus RNA. The transmembrane protease, serine 2 (TMPRSS2) inhibitors block the cellular entry of the SARS-CoV-II virus through the downregulated priming of the SARS-CoV-II spike protein. In order to prevent that an outbreak of respiratory sickness caused by a novel coronavirus (Covid-19) has become a serious public threat and disrupted many lives, assessing the efficacy of Zn-ejector drugs such as disulfiram combined with interferon to treat Covid-19 infected patients has been proposed. Zinc supplementation plus CQ/HCQ should be recommended for high risk or also younger patients outside of clinical trials as a prevention or treatment approach during SARSCoV-2 pandemic, should be considered only on a case-by-case basis. SARS coronavirus envelope protein ion channel activity promotes virus fitness and pathogenesis, in which $E$ protein ion channel activity represents a new determinant for SARS-CoV virulence. Transient zinc chelation (TPEN) induces ER stress and antiviral response by activating $N F-\kappa B$ leading to induction of interferon signaling and zinc plays divergent roles in rotavirus and dengue virus infections in epithelial cells. The antiviral compounds including zinc $N$-ethyl-N-phenyldithio-carbamate (EPDTC) inhibit the viral protease, thus preventing humancoxsackie-virus strain B3 (CVB3) genome replication. The interactions had been found on the binding specificity by $\mathrm{Zn}^{2+}$ ions-centered tetrahedral geometric coordination of the inhibitors against $3 C$ and $3 C$-like proteases.
\end{abstract}

Zinc-finger antiviral protein (ZAP) controls virus entry, DNA/RNA replication, and spreading against viral infection. ZAP specifically inhibits the replication of certain viruses and promotes viral RNA degradation that ZAP inhibits Retroviral RNA production and HIV-1 infection by promoting the degradation of specific viral mRNAs. The mutations of both protein and RNA at the RNA-ZAP interacting surface reduce the in vitro binding affinity and antiviral activity, in which ZAP coordination promotes downstream RNA degradation. ZAP specifically inhibits the replication of Moloney murine leukemia virus (MLV) and Sindbis virus (SIN) by preventing accumulation of the viral $m R N A$. The $m R N A$ decay is largely determined by the cis-acting elements. However, this ZAP's efficiency for COVID-19 remains yet unclear. Zinc-ions complexes as 2019$n C o V 3 C$-like protease inhibitors may play important role for this $\mathrm{Zn}^{2+}$-centered coordination pattern that the zinc-coordinating inhibitor is tetrahedrally coordinated to such as the catalytic dyad of CVB3 $3 C^{\text {pro }}$. Thus, $\mathrm{Zn}^{2+}$ ions-induced virucidal activities may function by viral destruction via cell surface receptor that $\mathrm{Zn}^{2+}$--virus interactions had been found on the binding specificity by $\mathrm{Zn}^{2+}$ ions-centered tetrahedral geometric coordination, functioning as the inhibitors.

Keywords: 2019-nCoV RNA mutation, Zinc chelation, ZAP and ZBD, RNA degradation, Zinc-coodinated inhibitor 
Abbreviations; $\mathbf{A D A R}=$ adenosine deaminases acting on RNA, ACE2=angiotensin-converting enzyme 2, APN=Amino-peptidase protein, ARDS=acute respiratory distress syndrome, $\mathbf{C o V s}=$ coronaviruses, COVID-19=coronavirus disease 2019, CQ/HCQ= chloroquine/ hydroxychloroquine. $\mathbf{C V B 3}=$ humancoxsackievirus strain B3, EPDTC=N-ethyl-Nphenyldithio-carbamate, $\quad$ FDA=food and drug administration, HCoV=human coronavirus, HR1= heptad repeat 1, IFITMs=Interferon induced transmembrane proteins, MERS-CoV=Middle East respiratory syndrome coronavirus, $\mathrm{MLV}=$ murine leukemia virus, $\mathbf{N A C}=\mathrm{N}$-acetylcysteine, $\mathbf{O R F}=$ open reading frames, 2019-nCoV=novel coronavirus 2019, $\mathbf{P C R}=$ polymerase chain reaction, $\mathbf{P P I}=$ protein-protein interaction, RCT=randomized controlled trial, $\mathbf{R d R p}=$ RNA-dependent RNA-polymerase, $\mathbf{R O S}=$ reactive oxygen species, $\mathbf{R S V}=$ respiratory syncytial virus, SARS-CoV=severe acute respiratory syndrome coronavirus, SARS-CoV-2= severe acute respiratory syndrome coronavirus $2, \quad \mathbf{S I N}=$ Sindbis virus, $\mathbf{S N P}=$ single nucleotide polymor-phisms, 6HB= six-helical bundle, TMPRSS2 $=$ transmembrane protease, serine 2, TPEN= N,N,N',N'-tetrakis (2pyridinyl-methyl)-1,2-ethanediamine, $\quad$ ZAPs=zinc finge $r$ antivirus proteins, TPEN= transient zinc chelation, ZNFs=zinc-finger proteins, UTR= untranslated region.

\section{INTRODUCTION}

After the first outbreak of coronavirus disease 19 COVID-19 infection, human-to-human transmission has accelerated the outbreak and case reports have started from other countries that coronaviruses are viruses whose genome structure is best known among all RNA viruses and prevention in this early stage of COVID-19 outbreak has been proposed that very recently, novel coronavirus (COVID-19) outbreak and quickly serious large spread worldwide caused by SARS-CoV-2 infection has posed a serious threat to global public health [1]. Human coronaviuses (HCoVs) are recognised as coronaviruses (CoVs) associated with multiple respiratory diseases of varying severity, including common cold, pneumonia and bronchilitis that to date, seventh known HCoVs have been identified, namely HCoVs-229E, HCoV-NL63, HCoV-OC43, HCoV-HKU1, severe acute respiratory syndrome coronavirus (SARS-CoV), Middle East respiratory syndrome coronavirus (MERS-CoV), and recently newtyped 2019-nCoV or SARS-CoV-2, coronavirus disease 19 COVID-19, subsequent phylogenetic studies pointed to the bat origin of SARS-CoV based on sequences of SARS-like virus found in bats [2]. This 2019-nCoV has single-stranded positive-sense RNA virus with 5'-cap and 3'-poly-A tail and the genome size of $30 \mathrm{~kb}$ which is the largest among all RNA viruses that caused a major outbreak of coronavirus disease 2019 COVID-19 and instigated a widespread fear and has threatened global health seculity [3]. Factor of present outbreak of disease 19 COVID-19 may be considered to be due to RNA virus mutation that the RNA viruses have high mutation rate and these high rates are correlated with enhanced virulence and evolvability, traits considered beneficial for viruses [4].

On the other hand, zinc( II ions play important role for these infectious diseases. Zinc is essential for highly growth and develop-ment of all organisms in the human body, especially the immune system that zinc has antiviral effects; it improves immune responses and suppresses viral replication. Zinc homeostatic status is a key factor in maintaining a healthy immune system. Zinc ions are involved in regulating intracellular signaling pathways in innate and adaptive immune cells that the influences of zinc status on the overall immune function are present in zinc defficiency as overproduction of pro-inflammatory cytokines and reactive mediators, zinc homeostasis as balanced immune cell functions, and zinc excess as suppression of $\mathrm{T}$ and $\mathrm{B}$ cell functions [5]. Zinc is a fundamental trace element in human body that the recommended daily intake of zinc depends on several factors. Average values of recommended intake may be $711 \mathrm{mg} /$ day for adults. Zinc is the second abundant trace metal with human body 2 $3 \mathrm{~g}$ and a plasma concentration of $12-16 \mu \mathrm{M}, 90 \%$ in muscle and bone, and $10 \%$ other organs include prostate, liver, the gastrointestinal tract, kidney, skin, lung brain, heart, and pancreas in humans that cellular zinc underlies an efficient homeostatic control that avoids accumulation of zinc in excess. Zinc status play an important role in antiviral immunity, mainly during the early stage of the infection that the most effective antiviral antibodies are neutralizing antibodies which bind to the viral envelope or capsid proteins, and regulate the virus entering into host cell [6]. In mammals, the plasma concentration of zinc ranges from 14 to 23 $\mu \mathrm{mol} / 1$ under normal physiological conditions, and serum zinc accounts for only $0.1 \%$ of the body's total zinc pool, $80 \%$ loosely bound by albumin and $20 \%$ bound by macroglobulin. Thus, sufficient daily intake of zinc is required to achieve steady state levels. In order to meet 
the daily requirement, the World Health Organization recommends a daily zinc intake of 9.4-10 $\mathrm{mg}$ and $6.5-7.1 \mathrm{mg}$ for men and women, respectively [7]. A variety of effects of zinc on immune cells depend on the zinc concentration. In a concentration of $100 \mu \mathrm{mol} / \mathrm{L}$, zinc suppresses natural killer cell killing and T-cell function whereas monocytes are activated direcly, and in a concentration of $500 \mu \mathrm{mol} / \mathrm{L}$, zinc evokes a direct chemotactic activation of neutrophil granulocytes [8]. $\mathrm{Zn}^{2+}$ ions have an important role for RNA viral destruction that the zinc-finger antiviral protein could regulate RNA virus degradation of SARS-CoV's and MERSCoV's RNA virus. Zinc ions can lead to RNA virus degradation and to viral death by a receptor-destroying enzyme [9]. Zinc ions become used as $\mathrm{Zn}^{2+}$-coordinated inhibitors for viral regulation of virucidal activities [10].

Furthermore, zinc-finger proteins (ZNFs) for health and disease have play an important role with DNA, RNA, PAR (poly-ADP-ribose) and ZFNs are involved in the regulation of several cellular processes such as transcriptional regulation, ubiquitin-mediated protein degradation, signal transduction, DNA repair, cell migration, and other processes [11]. Increasing the intracellular $\mathrm{Zn}^{2+}$ dose could capably damage the replication of a wide range of RNA viruses, such as poliovirus, influenza virus and SARS-CoV, and equine arteritis virus (EAV) [12]. Thus, zinc ions can prevent in the early stage of COVID-19 coronavirus outbreak.

In this review, firstly 2019-nCoV molecular structure and RNA virus mutation are described, secondly, $\mathrm{Zn}^{2+}$ ions-induced homeostatic immune infectious activities for preventative and antibody 2019-nCoV, and respiratory and pulmonary COVID-19 patient are dicussed, and lastly zinc-finger antiviral ptoteins (ZAPs) inhibit virus entry, RNA replication, spread, promote RNA virus degradation and generate ROS in respiratory and pulmonary COVID-19, in which the molecular mechanisms are clarified against 2019-nCoV infection.

\section{ZINC IMMUNITY IN INFECTION}

The innate immune system represents the defense first line against a pathogen before the adaptive system can develop the appropriate response. Many organs are affected by zinc deficiency, especially the immune system that is markedly susceptible to changes of zinc levels which the immune response involves in the regulation of the innate and adaptive immunity, and this zinc homeostasis is critical for sustaining proper immune function [13]. Thus, inflammation is a natural process required to protect the host from tissue damage and infections, which leads to the resolution of the inflammatory response and the restoration of homeostasis. Despite zinc deficiency can be treated by proper zinc intake, suboptimal zinc status cannot simply diagnosed by reason of the lack of clinical sighns and reliable biochemical indicators of zinc status. High zinc concentration is that zinc binding to proteins can activate or inactivate thir activity, or change characteristics important for substrate binding, whiles, zinc homeostasis is primarily controlled via the expression and action of 14 zinc tranporters that decreasing cytoplasmic zinc can describe export via ZnTs, but also the transport of zinc into one of those organelles [14]. Zinc homeostasis during acute phase response is the temporal transfer of serum zinc to the tissues, causing transient serum hypozincemia, which is rebalanced during resolution of the inflammatory response that intracellularly increased zinc can intoxicate engulfed pathogens and acts cytoprotective by promotion of neutralizing reactive oxygen species (ROS) and nitrogen species (RNS) [14].

2019-NCOV SPIKE, MEMBRANE, ENVELOPE, AND NUCLEOCAPSID PROTEINS, AND 2019NCOV RNA MUTATION

Coronaviruses (CoVs) are viruses whose genome structure is best known among all RNA viruses that typical $\mathrm{CoV}$ genome contains at least six open reading frames (ORFs) which the first ORF $(\mathrm{ORF} 1 \mathrm{a} / \mathrm{b})$ is about two-thirds of the whole genome length encodes 16 non-structural proteins, in which ORFs near $3^{\prime}$ end of the genome encodes at least four main structural proteins including spike (S), membrane $(\mathrm{M})$, envelope $(\mathrm{E})$, and nucleocapsid $(\mathrm{N})$ proteins [15]. Most of the non-structural proteins have encodes viral polymerase, RNA-dependentRNA-polymerase (RdRp). CoVs are enveloped, spherical or pleiomophic viruses, with typical sizes ranging from 80 to $120 \mathrm{~nm}$ that they possess a 5' capped, single-strand positive sense RNA genome with a length between 26.2 and $31.7 \mathrm{~kb}$. 2019-nCoV makes use of a densely glycosylated $S$ protein to gain entry into host cells. The $\mathrm{S}$ protein is a trimeric class I fusion protein that exists in a metastable prefusion conformation that undergoes a substantial structural rearrangement to fuse the viral membrane with host cell membrane. Spike is 
cleaved into S1 and S2 by the host cell protease that the main function of $\mathrm{S} 1$ is to bind ewith the host cell surface receptors, and the S2 submit mediates virus-cell and cell-cell membrane fusion, Hence, the therapeutic stragies to block coronavirus from entering host cells by spike proteins or specific receptors on the host surface may be valuable for the antiviral development [16]. The ionophore in this RNA virus makes the cell membrane porous to the zinc ion that $\mathrm{Zn}^{2+}$ ions could inhibit virus entry and membrane fusion of $\mathrm{S} 1$ and $\mathrm{S} 2$ domains of Spike Protein with zinc ion-binding interaction. This process is triggered when the S1 submit binds to a host cell receptor, and receptor binding destabilizes the prefusion trimer, resulting in shedding of the S1 subunit and transition of the S2 subunit to a stable postfusion conformation [17].

The $\mathrm{CoV} \mathrm{E}$ protein is a small, integral membrane protein involved in several aspects of the virus' life cycle that the most progress has been made on SARS-CoV E, highlighting specific structural requirements for its functions in the $\mathrm{CoV}$ life cycle as well as mechanism behind its pathogenesis [18]. The typical CoV genome includes a 5'-cap, 5'-untranslated region (UTR), open reading frames, a 3'-UTR, and 3'-poly (A) tail. In addition, 2019-nCoV has a unique four amino acid insertion between $\mathrm{S} 1$ and S2 domains of the spike protein, which created a potential furin or TMPRSS2 cleavage site. 2019-nCoV may increase its infectivity through the receptor binding domain recombination and a cleavage site insertion [19].

Mutations and adaptation in the $\mathrm{S}$ and $\mathrm{N}$ genes could affect virus stability and pathogenicity. As more genomes are made publicly available, analysis of the genome sequence diversity across samples has revealed the highest diversity occurring in the structural genes, especially the $\mathrm{S}$ protein, ORF3a, and ORF8 [20]. Thus, the major mutations are in the critical proteins, including the $\mathrm{S}$ protein, RNA polymerase, RNA primase, and nucleoprotein [21]. This nove Yinl coronavirus (2019-nCoV) outbreak has caused a global pandemic resulting many infected persons and deaths worldwide that the RdRp catalyzed the synthesis of viral RNA, is a key component of coronaviral replication/transcription as a primary targeted antiviral drug [22]. It is unclear whether zinc ions can suppress RNA mutation and outbreak by RNA mutation.

\section{The Role of Zinc Ions on Prevention AND ANTIBODY Against Covid-19 INFECTION}

Enhancement of zinc immunity for preventing infection with the coronavirus SARS-CoV-2 that causes COVID-19 are urgently needed. For trials to prevent infection with human immunodeficiency virus, in which serological measurements at baseline and during and after the trial are routine. On the other hand, trials of prophylactic drugs or physical prophylaxis are often performed for infections, such as infection with the malaria pathogen Plasmodium falciparum or with influenza virus, respectively. For this reason, baseline testing has been variable. Clinical trials are being set up at a rapid rate to test various approaches to preventing COVID-19. While accurate serological tests are still in development, trialists have a window of opportunity for obtaining blood from trial participants and banking it in anticipation of having such tests in the near future [23]. Of clinical importance, such impaired antibody-mediated responses could be restored by zinc supplementation. Higher intracellular zinc concentration has shown to increase monocyte resistance to apoptosis via suppressing the activation of caspase, $50 \mathrm{mg}$ of zinc per day might provide an additional shield against the COVID-19 pandemic, possibly by increasing the host resistance to viral infection to minimize the burden of the disease. As mentioned, the potential beneficial role of zinc in COVID-19 infection needs further clinical validation, however, in this pandemic situation, using zinc to reduce disease burden would be a well-intentioned trial [24, 25].

Zinc induced preventative antibody that neutralizes SARS-CoV-2 binds a conserved epitope on the spike receptor binding domain explaining its ability to cross-neutralize SARS$\mathrm{CoV}$ and SARS-CoV-2, using a mechanism that is independent of receptor binding inhibition. This antibody will be useful for development of antigen detection tests and serological assays targeting SARS-CoV-2. Neutralizing antibodies can alter the course of infection in the infected host supporting virus clearance or protect an uninfected host that is exposed to the virus. Hence, this antibody offers the potential to prevent and/or treat COVID-19, and possibly also other future emerging diseases in humans caused by viruses from the Sarbecovirus subgenus [26]. In addition to their viral receptor functions, the receptors for coronaviruses have 
their own physiological functions angiotensinconverting enzyme 2 (ACE2) is a zincdependent carboxypeptidase that cleaves one residue from the $\mathrm{C}$ terminus of angiotensin peptides and functions in blood pressure regulation. ACE2 also protects against severe acute lung failure, and SARS-CoV-induced downregulation of ACE2 promotes lung injury.Amino-peptidase protein (APN) is a zincdependent aminopeptidase that cleaves one residue from the $\mathrm{N}$-terminus of many physiological peptides and plays multifunctional roles such as in pain regulation, blood pressure regulation, and tumor cell angiogenesis. Sugars decorate many proteins and fats on cell surfaces and function in many biological processes such as immunity and cell-cell communi-cation. How these cell-surface molecules are selected by viruses as their entry receptors has been a major puzzle in virology [27].

Zinc is known to modulate antiviral and antibacterial immunity and regulate inflammatory response that the individual preventive and protective measures drive the personal risk of getting the disease. Zinc ions inhibit the RNAdependent RNA polymerase, which crucially replicates copies of viral RNA in the host cells. Remdesivir inhibits coronavirus with the intact proofreading, thus renders its superior antiviral efficacy. Zn status and respiratory syncytial virus (RSV) infection. Particularly, whole blood zinc was signifi-cantly lower in children with RSV pneumonia and $\mathrm{Zn}$ compounds were shown to inhibit respiratory syncytial virus replication and RSV plaque formation with a more than 1,000fold reduction at $10 \mu \mathrm{m} \mathrm{Zn}$ preincubation. Thus, $\mathrm{Zn}$ may possess protective effect as preventive and adjuvant therapy of COVID-19 through reducing inflammation, improve-ment of mucociliary clearance, prevention of ventilator-induced lung injury, modulation of antiviral immunity [28].

Evidence for vitamins C, D and zinc and their roles in preventing pneumonia and respiratory infections (vitamins C and D) and reinforcing immunity (zinc) appears to look particularly promising. Tolerable upper intake levels (ULs) are intake levels which should not be surpassed as toxicity problems could appear. For vitamin $\mathrm{D}$ a UL of $50 \mu \mathrm{g} /$ day is advised and for zinc a UL of $25 \mathrm{mg} /$ day is recommended. supplemental daily doses of up to about $1 \mathrm{~g}$, in addition to normal dietary intake, are not associated with adverse gastrointestinal effects Zinc is also involved in inflammation, elevating inflammatory responses and inducing cell- mediated immunity, and is a key component of pathogen-eliminating transduction pathways that contribute to neutrophil extracellular traps (networks which bind pathogens) formation [29].

ZinC IONS-Mediated VIRUCIDAL ACTIVITIES IN RESPIRATORY AND PULMONARY 2019-NCOV INFECTION

Clinical features associated with patients infected with SARS-CoV, MERS-CoV and SARS-CoV-2 range from mild respiratory illness to severe acute respiratory disease. Both MERS and SARS patients in later stages develop respiratory distress and renal failure Pneumonia appears to be the most frequent manifestation of SARS-CoV-2 infection, characterized primarily by fever, cough, dyspnea, and bilateral infiltrates on chest imaging that the period from infection to appearance of symptoms varies. Generally, it is thought to be days, however, a research group at Guangzhou Medical University reported the incubation period to be 24 days. In a family cluster of infections, the onset of fever and respiratory symptoms occurred approximately three to six days after presumptive exposure [29 30]. SARS-CoV-2 enters the target cells through the angiotensin-converting enzyme 2 (ACE2) receptor and the transmembrane protease, serine 2 (TMPRSS2). The TMPRSS2 inhibitors block the cellular entry of the SARSCoV-II virus through the downregulated priming of the SARS-CoV-II spike protein [31]. $\mathrm{Zn}^{2+}$ inhibits coronavirus and anterivirus RNA polymelase activity, and zinc ionophores block the virus replication that $\mathrm{Zn}^{2+}$ and pyrithione at low concentrations inhibit the replication of SARS-CoV and arterivirus RNA [32]. The other, high zinc ion concentration and the addition of compounds that stimulate cellular zinc ions were found to inhibit the replication of various RNA virus, influenza viruses, respiratory syncytial virus and coronaviruses [32].

In order to prevent that an outbreak of respiratory sickness caused by a novel coronavirus (Covid-19) has become a serious public threat and disrupted many lives,assessing the efficacy of FDA-approved Zn-ejector drugs such as disulfiram combined with interferon to treat Covid-19 infected patients has been proposed. Based on evolutionary and physical principles of the key factors controlling the reactivity of Zn-bound Cys, having identified putative labile $\mathrm{Zn}$-sites in Covid-19 that can be targeted by $\mathrm{Zn}$-ejector drugs, leading to $\mathrm{Zn}^{2+}$ 
release and viral structure/function disruption. It presents an avenue for treating Covid19-infected patients using clinically safe Zn-ejecting drugs to attack conserved catalytic and/or $\mathrm{Zn}$ bound cysteines in multiple targets; thus, assessing their efficacy combined with interferon in clinical settings would be of great interest. Our strategy based on evolutionary and physical principles is general and can be used to identify druggable $\mathrm{Zn}$-sites in conserved domains of other viruses. Importantly, it offers a possible strategy to tackle future outbreaks of pandemic viruses: FDA-approved drugs for a certain conserved domain may be repurposed to target the same conserved domain found in a new infectious virus. Furthermore, by targeting conserved domains with druggable $\mathrm{Zn}$-sites, drugs may be used to treat several types of viruses [33]. Parenteral zinc + chloroquine /hydroxychloroquine (CQ/ HCQ) in the treatment of hospitalized COVID-19 patients may help to improve clinical outcomes and to limit the COVID-19 fatality rates. Therefore, whether zinc supplementation in combination with CQ/HCQ should be recommended for high risk or also younger patients outside of clinical trials as a prevention or treatment approach during SARS-CoV-2 pandemic, should be considered only on a case-by-case basis $[\mathbf{3 4}, \mathbf{3 5}]$

SARS coronavirus envelope protein ion channel activity promotes virus fitness and pathogenesis that inflammasome-activated IL- $1 \beta$ levels were reduced in the lung airways of the animals infected with viruses lacking $\mathrm{E}$ protein ion channel activity and acute respiratory distress syndrome (ARDS) leading to death, in which E protein ion channel activity represents a new determinant for SARS-CoV virulence [36].

On the case of preventing lung and pulmonia, firstly, 2019-nCoV nucleic acid detection is carried out that accurate RNA detection of 2019-nCoV is with diagnostic value (Strong recommendation). The RNA of 2019-nCoV positive in the throat swab sampling or other respiratory tract sampling by fluorescence quantitative polymerase chain reaction (PCR) method, especially that from multiple samples and detection kits, excluding sample quality, sample collection time, contaminatory and technical problems, is of great support for etiological diagnosis. Drug treatment; (1)At present, there is no evidence from randomized controlled trial (RCT) to support specific drug treatment against the new coronavirus in suspected or confirmed cases. (2) The $\alpha$ - interferon atomization inhalation can be considered ( 5 million $U$ per time for adults in sterile injection water, twice a day) (Weak recommendation); lopinavir/ritonavir orally, 2 capsules each time, twice a day, can be also considered (Weak recommendation). As antibiotic therapy; (1) Principles. Avoid blind or inappropriate use of antibacterial drugs, especially the combination of broad-spectrum antibacterial drugs. Enhancement of bacteriological surveillance should be performed and promptly given appropriate antibacterial drugs when it occurs secondary bacterial infection. (2) According to the clinical manifestations of patients, if the accompanying bacterial infection cannot be ruled out, mild patients can take antibacterial drugs against community-acquired pneumonia, such as amoxicillin, azithromycin, or fluoroquinolones; empirical antibacterial treatment in severe patients should cover all possible pathogens, deescalating therapy until the pathogenic bacteria are clarified [37].

Preventions against lung damages include avoiding direct lung infection, altering hostvirus interactions, promoting immune responses, diluting virus concentrations in lung tissues by promoting viral migration to the rest of the body, maintaining waste removal balance, protecting heart function and renal function, avoiding other infections, reducing allergic reactions and anti-inflammatory. This treatment protocol using water, air, salt, sound, temperature, emotion, exercise, etc. can be the most powerful cures for viral and non-viral lung infections because they do not depend on molecular specificity and are freely available to anyone

The first purpose is avoiding exposures that could result in widespread damages to lungs and taking post exposure mitigating measures that would reduce disease severity. The second purpose is reducing death rate and disability rate from the current levels to one tenth for infected patients by using multiple factors health optimization method. The double reduction objects are expected to generate a series of chain reactions that favor mitigating or ending the pandemic [38].

Improve lung micro circulation to prevent damages to the lungs, Vitamins and essential nutrients for the immune system (but not for the virus) may shorten the phase lag by one to two days and thus make a difference; deep breaching can improve energy metabolism by as much as $30 \%$ (for experienced, it may improve more); and avoiding exercise may save MET values by 
Zinc( II) Immune Virucidal Activities for 2019-nCoV Prevention and COVID-19 Respiratory Ailment and Pneumonia

up to $70 \%$; relaxation exercise can reduce blood circulation by $10 \%$ to $30 \%$; avoiding a secondary infection can reduce burden on the immune system, reduce viral burden on lungs, kidneys and heart, and help maintain the waste balance in the lungs. Warn foods such as ginger, date, citrus, etc are known to improve blood circulation and energy production [39].

However, Zinc supplementation did not yield a statistically significant reduction in symptoms in children with severe pneumonia. Zinc supplements given during an acute episode are not beneficial in short-term clinical recovery from severe pneumonia in hospitalized children $[\mathbf{4 0 , 4 1 ]}$.

Transient zinc chelation N,N,N',N'-tetrakis(2pyridinylmethyl)-1,2-ethanediamine (TPEN) led to induction of an antiviral state that in cells via induction of heat shock proteins and activation of NF- $\mathrm{KB}$ and up regulation of downstream effectors which inhibit DENV replication. Interferon-stimulated genes (ISGs) are a large group of genes which have diverse effects on viral infections and mostly act at early stages of virus life-cycle. Therefore, cellular or tissue zinc homeostasis may also determine the efficiency with which pathogens replicate and disseminate in vivo. In the case of acute viral infections, strategies to transiently block zinc redistribution during viremic stages may inhibit viruses that depend on cellular zinc pools for replication. This would provide a window for the immune system to gain an upper hand and control viral infection. Zinc chelation abrogated dengue virus RNA replication and zinc chelation abrogated dengue virus RNA replication. Transient zinc chelation induces ER stress and antiviral response by activating NF-kappaB leading to induction of interferon signaling and zinc plays divergent roles in rotavirus and dengue virus infections in epithelial cells [42]. Zinc-coordination pattern; the antiviral compounds including zinc N-ethyl-Nphenyldithiocarbamate (EPDTC), inhibit the viral protease, thus preventing human coxsackie virus strain B3 (CVB3) genome replication. The interactions had been found on the binding specificity by $\mathrm{Zn}^{2+}$ ions-centered tetrahedral geometric coordination of the inhibitors against $3 \mathrm{C}$ and $3 \mathrm{C}$-like proteases [43].

Thus, $\mathrm{Zn}^{2+}$ ions-induced virucidal activities may be due to viral destruction via cell surface receptor that $\mathrm{Zn}^{2+}$ - virus interactions had been found on the binding specificity by $\mathrm{Zn}^{2+}$ ions-centered tetrahedral geometric coordination, functioning as the inhibitors.

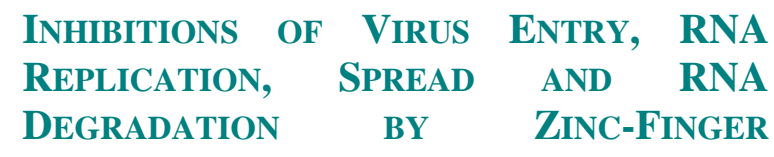
Antiviral Protein

The zinc-finger antiviral protein (ZAP) controls virus entry, DNA/RNA replication, and spreading against viral infection. The ZAP in first steps of HCV infection may be used as entry inhibitor [44]. Interferon induced transmembrane proteins (IFITMs) inhibit the cellular entry of a broad range of viruses that IFITM-mediated restriction requires recognition of viral RNA elements [45]. The interferonstimulated genes serve as enhancers of antiviral innate immunity [46]. ZAP inhibits alphavirus replication that elucidation of the antiviral mechanism by which ZAP inhibits Sindbis virus (SINV) translation may lead to the development of agents with broad activity against alphaviruses [47]. The ZAP also inhibits Influenza A virus (IAV) protein expression, in which suggests an important role of ZAP in the host effort to control IAV infection and the importance of the threat of ZAP to the virus [48]. The host cell restriction factors that limit IAV have been investigated [49]. ZAP may regulate DNA and RNA virus replication. Inhibition of bacterial DNA replication during nitrosative stress is accompanied by zinc mobilization [50].

Zn72D both 20 regulates adenosine deaminases acting on RNA (ADAR) protein levels and interacts with ADAR in an RNA- dependent fashion, and similar to ADAR, Zn72D is necessary to maintain proper neuromuscular junction architecture and motility in the fly. Furthermore, the mammalian homolog of Zn72D, Zfr, regulates editing in mouse primary neurons, demonstrating the conservation of this regulatory role. The broad and conserved regulation of $\mathrm{ADAR}$ editing by $\mathrm{Zn} 72 \mathrm{D}$ in neurons represents a novel mechanism by which critically important editing events are sustained [51].

ZBD could regulate 2019-nCoV RNA spike that zinc-binding status having $\mathrm{Zn}^{2+}$ ions-centered coordination structure could serve as the development of potential drugs for SARS therapies. A complex zinc finger ZBD modulates the enzymztic activities of coronaviridaeNidovirus helicases, leading that the ZBD is critically involved in nidovirus replication and trascription [52]. 


\section{ZAP REGULATES SPREAD}

ZAP' stress with antiviral activity and induced virus replication are regulated upon virus infection to inhibit virus spread [53]. ZAP-70 kinase regulates HIV cell-to-cell spread that HIV usurps components of the immunnological synapse machinery to ensure its own spread through cell-to-cell contacts [54]. An understanding of viral cell-to-cell transmission spreading will enhance our ability to intervene in the efficient spreading of viral infection [55].

\section{RNA DEGRADATION}

ZAP specifically inhibits the replication of certain viruses and promotes viral RNA degradation [56]. ZAP inhibits Retroviral RNA production [57] and ZAP inhibits HIV-1 infection by promoting the degradation of specific viral mRNAs [58]. The four zinc fingers of ZAP form extensive interactions with RNA, but mutations of both protein and RNA at the RNA-ZAP interacting surface reduce the in vitro binding affinity and antiviral activity, in which ZAP coordination promotes downstream RNA degradation [59].

ZAP is a host antiviral factor that specifically inhibits the replication of Moloney murine leukemia virus (MLV) and Sindbis virus (SIN) by preventing accumulation of the viral mRNA in the cytoplasm. The mRNA degradation or the mRNA decay is largely determined by the cisacting elements. ZAP directly interacted with the exosome component and that the binding region of ZAP was mapped to amino acids 224254. Depletion of the exosome component with small interfering RNA significantly reduced ZAP's destabilizing activity which ZAP is a trans-acting factor that modulates mRNA stability [60].

\section{ZINC INDUCED ROS GENERATION IN RESPIRATORY AND PULMONARY COVID- 19 INFECTED CELLS}

Zinc induced ROS generation in respiratory and pulmonary COVID-19 infected cells is that the univalent reduction of oxygen generates superoxide $\left(\cdot \mathrm{O}_{2}{ }^{-}\right)$, hydrogen peroxide $\left(\mathrm{H}_{2} \mathrm{O}_{2}\right)$, and hydroxyl radicals $(\bullet \mathrm{OH})$, all of which are reactive oxygen species (ROS). Superoxide has an unpaired electron, which imparts higher reactivity and renders it very unstable and shortlived. ROS are usually produced continuously in vivo under aerobic conditions. The production of ROS and its elimination by the anti-oxidant defense system in cells is a highly modulated process for maintaining normal physiological function in the body, in which the nicotinamide adenine dinucleotide phosphate (NADPH) oxidases are a group of plasma membraneassociated enzymes which catalyze the production of superoxide $\cdot \mathrm{O} 2$ - from oxygen by using NADPH as the electron donor [61].

In respiratory COVID-19 infected cells is that Respiratory viruses are known to induce ROSgenerating enzymes, including nicotinamide adenine dinucleotide phosphate oxidases (NADPH oxidases, Nox) and xanthine oxidase (XO) and to disturb antioxidant defenses. Increased activities of the Nox and Dual oxidase (Duox) family were observed both in vitro and in vivo. So far, no efficient measures for prevention and treatment exist, in which changes in redox homeostasis in infected cells are one of the key events that is linked to infection with respiratory viruses and linked to inflammation and subsequent tissue damage [62]. The other, Zinc induced ROS generation in pulmonary COVID-19 infected cells is that Alterations of ROS-producing and scavenging pathways that are caused by respiratory viral infections are implicated in inflammation, lung epithelial disruption, and tissue damage, and, in some cases, even pulmonary fibrosis. These events are at least partially interregulated: inflammation can contribute to lung damage and epithelial dysfunction and vice versa. The observation that ROS are implicated in the pathology of these viruses is mainly based on experimental infection models. Such inflammatory processes, especially sustained chronic conditions of inflammation, along with inflammation-induced oxidative stress from dead or injured cells, could lead to irreversible cell ularortissue damage with the passage oftime, which further contributes to the development of chronic degenerative diseases [62]. The role of excessive immune activation as the cause of lung destruction by SARS-CoV-2 supposes the causative virus of the pandemic coronavirus disease 19 (COVID19). The oxidative stress by virally induced ROS production spirals cytokine release and immune cell infiltration in the lung as a result. To handle the toxic ROS response it would be of value to evaluate the administration of NAC in preclinical and clinical trials as a potentially costeffective intervention for virus-infected patients with symptoms of lung dysfunction, with the aim to normalize the redoxhomeostasis. The administration route should also be evaluated prior a trial start, as both oral 
infusion as well as inhalation administration routes are available. NAC has also been show to interact and inhibit proteasome inhibitors, it remains to be shown if NAC or other pharmacological agents can affect virally released proteasome binding proteins and thereby aid proteasome function and by this, prevent tissue damage [63].
As mentioned above, $\mathrm{Zn}^{2+}$ ions induced virucidal activities for prevention and antibody, respiratory ailment and pulmonary disease against COVID-19 infection are represented in Table 1. However, COVID-19 degradation or destruction by zinc-finger antiviral proteins remains yet unclear and COVID-19 pulmonary care by zinc ion solutions may be of importance.

Table1. $\mathrm{Zn}^{2+}$ ions-induced virucidal activities for prevention and antibody, respiratory ailment and pulmonary disease against COVID-19 infection

\begin{tabular}{|c|c|c|c|}
\hline $\begin{array}{l}\mathrm{Zn}^{2+} \\
\text { ions }\end{array}$ & \multicolumn{3}{|c|}{$\begin{array}{l}\mathrm{Zn}^{2+} \text { ions induced antiviral activites for prevention, and repiratory } \\
\text { ailment and pulmonary disease against COVID-19 infection }\end{array}$} \\
\hline \multirow[b]{2}{*}{$\mathbf{Z n}^{2+}$} & $\begin{array}{l}\text { Prevention and } \\
\text { antibody }\end{array}$ & Respiratory infection & Pulmonary inflammation \\
\hline & $\begin{array}{l}\rightarrow \quad \mathbf{Z n}^{2+} \\
\cdot \text { Zn homeostatic } \\
\text { immune conc 50 } \\
\text { mg/day } \\
\cdot \text { Zinc supplementation } \\
\text { in combination } \\
\text { with CQ/HCQ } \\
\cdot \text { Zinc supplement } \\
\text { prevents pneumonia } \\
\text { in children } \\
\cdot \text { TRPV1 prevention } \\
\cdot \text { Lower } \mathbf{Z n}^{2+} \text { conc may } \\
\text { be efficient for vaccine } \\
\text { candidate and higher } \\
\text { Zn' }{ }^{2+} \text { conc may prevent } \\
\text { respiratory ailment } \\
\text { and acute pneumonia } \\
\text { spreading against } \\
\text { HCoVs }\end{array}$ & $\begin{array}{l}\rightarrow \mathrm{Zn}^{2+}, \cdot \mathrm{O}_{2}^{-}, \mathrm{H}_{2} \mathrm{O}_{2}, \\
\cdot \mathrm{OH} \\
\cdot 2 \mu \mathrm{M} \mathrm{Zn}{ }^{2+}+2 \mu \mathrm{M} \text { Pyrithione } \\
(\mathrm{PT}) \text { inhibit RNA replication } \\
\cdot \text { Higher } \mathrm{Zn}^{2+} \text { conc }+\mathrm{HK} \text { inhibit } \\
\text { virus entry against DV } \\
\cdot \text { Zinc chelation inhibits RNA } \\
\text { replication } \\
\cdot \text { TMPRSS2 blocks cellular } \\
\text { entry } \\
\cdot \text { FDA-approved Zn-ejector } \\
\text { drugs such as disulfiram } \\
\cdot \text { ADAR-mediated RNA editing } \\
\cdot \text { 2019-nCoV RNA degradation } \\
\text { by Zn }{ }^{2+} \text { ions? } \\
\cdot \mathrm{ZnOTs} \mathrm{inhibit} \mathrm{HSV-1} \mathrm{entry} \\
\text { and spread } \\
\cdot \text { 2,500mg/kg diet } \mathrm{ZnO} \text { has } \\
\text { antiviral activity of SARS-CoV } \\
\cdot \mathrm{ZnONPs} \text { inhibit H1N1 } \\
\text { influenza virus entry }\end{array}$ & $\begin{array}{l}\rightarrow \mathrm{Zn}^{2+}, \cdot \mathrm{O}_{2}^{-}, \mathrm{H}_{2} \mathrm{O}_{2}, \cdot \mathrm{OH} \\
\cdot \text { CQ/HCQ plus zinc inhibit } \\
\text { RNA replication } \\
\cdot \text { Zinc-coordinated inhibitor } \\
\cdot \text { Zinc }+ \text { chloroquine } \\
\cdot \text { Zn-ejectors + disulfiram } \\
\cdot \text { ADAR-mediated RNA editing } \\
\text { targets } \\
\cdot \text { RNA degradation by zinc ions ? } \\
\cdot \text { Zinc-binding ACE2 ? } \\
\cdot \text { ZnONPs regulate microRNA in } \\
\text { Ovarian granulosa cells } \\
\cdot \text { ZnONPs + DMN inhibit the } \\
\text { production of mRNA of } \\
\text { inflammatory cytokines } \\
\cdot \text { ZAP degrades SARS-CoV's and } \\
\text { MERS-CoV's RNA } \\
\cdot \text { Complex zinc-finger inhibits } \\
\text { nidovirus replication }\end{array}$ \\
\hline
\end{tabular}

\section{Conclusions}

Recently new-typed 2019-nCoV or SARS-CoV2, coronavirus disease 19 COVID-19, subsequent phylogenetic studies pointed to the bat origin of SARS-CoV based on sequences of SARS-like virus found in bats. This 2019nCoV, SARS-CoV-2, or coronavirus disease COVID-19 have single-stranded positive-sense RNA virus with 5'-cap and 3'-poly-A tail and the genome size of $30 \mathrm{~kb}$ which is the largest among all RNA viruses that caused a major outbreak of COVID-19 and instigated a widespread fear and has threatened global health seculity. The CoV genome contains at least six ORFs which the first ORF1a/b is about twothirds of the whole genome length encodes 16 non-structural proteins, in which ORFs near 3' end of the genome encodes at least four main structural proteins including $\mathrm{S}, \mathrm{M}, \mathrm{E}$, and $\mathrm{N}$ proteins. But, most of the non-structural proteins are known to play a vital role in $\mathrm{CoV}$ replication and the structural proteins are important for virion assembly as well as for causing $\mathrm{CoV}$ infection, and two-thirds of RNA they have encodes viral polymerase, RdRp, RNA synthesis materials, and two large nonstructural polyproteins that are not involved in host response modulation of ORF1a-ORF1b.

Factor of present outbreak of disease 19 COVID-19 may be considered to be due to RNA virus mutation that the RNA viruses have high mutation rate and these high rates are correlated with enhanced virulence and evolvability, traits considered beneficial for viruses. However, it is unclear whether $\mathrm{Zn}^{2+}$ ions reduce the high mutation rate of RNA genome structure. 
The coronavirus spike glycoprotein $\mathrm{S}$ is a multifunctional molecular machine that mediates coronavirus entry into host cells.

It first binds to a receptor on the host cell surface through its spike protein S1 subunit and then fuses viral and host membranes through its spike protein S2 subunit. Two domains in S1 from different corona viruses recognize a variety of host receptors, leading to viral attachment. The spike protein exists in two structurally distinct conformations, prefusion and post fusion. The transition from prefusion to postfusion conformation of the spike protein must be triggered, leading to membrane fusion.

$\mathrm{Zn}^{2+}$ ions could inhibit virus entry and membrane fusion of $\mathrm{S} 1$ and $\mathrm{S} 2$ domains of Spike Protein with zinc ion-binding interaction. Whereas, the $\mathrm{M}$ and $\mathrm{E}$ proteins are involved in virus assembly, the spike glycoprotein is the leading mediator of viral entry that the spike protein is also the principal player in determining host range, and the spike protein plays a dual role in entry by mediating receptor binding and membrain fusion that the fusion process involves large conformational changes of the spike protein. 2019-nCoV may have similar membrane fusion mechanism as that of SARS-CoV. When S1 protein recognizes its receptor on human cells, the HR1 and HR2 domains are exposed to interact with each other, forming 6-HB to mediate membrane fusion between virus and target cell. Notably, both 2019-nCoVHR2P and EK1, the pan-CoV fusion inhibitor, exhibited potent inhibitory activity against S-mediated cell-cell fusion and 2019$\mathrm{nCoV}$ pseudovirus infection, suggesting potential development of either 2019-nCoVHR2P or EK1 peptide in nasal spray and inhalation formulations, respectively, to prevent and treat 2019-nCoV infection. and fusion inhibitors are targeting HR1 domain in spike protein.

Zinc ions can prevent in the early stage of 2019$\mathrm{nCoV}$ infected patient, and the zinc ions have important roles for respirarory and pulmonary process of COVID-19 disease. $\mathrm{Zn}^{2+}$ inhibits coronavirus and anterivirus RNA polymelase activity, and zinc ionophores block the virus replication that $\mathrm{Zn}^{2+}$ ions + pyrithione at low concentrations inhibits the replication of SARS$\mathrm{CoV}$ and arterivirus RNA. TMPRSS2 inhibitors block the cellular entry of the SARS-CoV-II virus through the downregulated priming of the SARS-CoV- II spike protein. In order to prevent that an outbreak of respiratory sickness caused by a novel coronavirus (Covid-19) has become a serious public threat and disrupted many lives,assessing the efficacy of FDA-approved Zn-ejector drugs such as disulfiram combined with interferon to treat Covid-19 infected patients has been proposed. Whether zinc supplementation $+\mathrm{CQ} / \mathrm{HCQ}$ should be recommended for high risk or also younger patients outside of clinical trials as a prevention or treatment approach during SARS-CoV-2 pandemic, should be considered only on a caseby-case basis. SARS coronavirus envelope protein ion channel activity promotes virus fitness and pathogenesis, in which E protein ion channel activity represents a new determinant for SARS-CoV virulence. Transient zinc chelation TPEN induces ER stress and antiviral response by activating NF- $\mathrm{KB}$ leading to induction of interferon signaling and zinc plays divergent roles in rotavirus and dengue virus infections in epithelial cells. The antiviral compounds including zinc N-ethyl-Nphenyldithio-carbamate (EPDTC) inhibit the viral protease, thus preventing humancoxsackievirus strain B3 (CVB3) genome replication. The interactions had been found on the binding specificity by $\mathrm{Zn}^{2+}$ ions-centered tetrahedral geometric coordination of the inhibitors against 3C and 3C-like proteases.

ZAP controls virus entry, DNA/RNA replication, and spreading against viral infection. ZAP specifically inhibits the replication of certain viruses and promotes viral RNA degradation. ZAP inhibits Retroviral RNA production and ZAP inhibits HIV-1 infection by promoting the degradation of specific viral mRNAs. The four zinc fingers of ZAP form extensive interactions with RNA, but mutations of both protein and RNA at the RNA-ZAP interacting surface reduce the in vitro binding affinity and antiviral activity, in which ZAP coordination promotes downstream RNA degradation. ZAP directly interacted with the exosome component and that the binding region of ZAP was mapped to amino acids 224-254. Depletion of the exosome component with small interfering RNA significantly reduced ZAP's destabilizing activity which ZAP is a transacting factor that modulates mRNA stability. However, this ZAP's efficiency for COVID-19 remains yet unclear. Zinc-ions complexes as 2019-nCoV 3C-like protease inhibitors may play important role for this $\mathrm{Zn}^{2+}$-centered 
coordination pattern that the zinc-coordinating inhibitor is tetrahedrally coordinated to the $\mathrm{His}^{40}{ }^{4} \mathrm{Cys}^{147}$ catalytic dyad of CVB3 $3 \mathrm{C}^{\mathrm{pro}}$.

\section{REFERENCES}

[1] A. Erdogan, P. M. Agaoglu, et al; 2019 novel coronavirus (COVID-19) outbreak: A review of the current literature. EJMO 2020;4(1):1-7.

[2] Y. X.Lim, Y. L.Ng, J. P. Tam and D.X. Liu (2016); Human coronaviruses: A review of virus-host interactions. Diseases. 4: 1-28 pages.

[3] M. U. Mirza and M. Froeyen; Structural elucidation of SARS-CoV-2 vital proteins: computational methods reveal potential drug candidates against Main protease, Nsp12 RNAdependent RNA polymerase and Nsp13 helicase. Preprint. 2020; 5 March: 2-23.

[4] S. Duffy; Why are virus mutation rates so damn high. PLOS Biology. 2018; 16(8): 1-6.

[5] I. Wessels, M. Maywald and L. Rink; Zinc as gatekeeper of immune function. Nutrients. 2017; 9(12): 1-44 pages.

[6] Hong Gao, Wei Dai, Lu Zhao, Junxia Min, et al; The Role of Zinc and Zinc Homeostasis in Macrophage Function. Journal of Immunology Research 2018 ;Volume 2018, Article ID 6872621, 11 pages https://doi.org/10.1155/20 $18 / 6872621$

[7] K-H. Ibs and L. Rink; Immunity enhanced by trace elements. J. Nutr. 2003; 133: 1452S-1456S.

[8] S. A. Read, S. Obeid, C. Ahlenatiel, G. Ahlenstiel; The role of zinc in antiviral immunity. Adv Nutr. 2019; 0:1-15.

[9] T. Ishida; Zinc Immune Anti-Infectious Activities of Bacteriolysis by $\mathrm{Zn}^{2+}$-induced Bacterial PGN Autolysins and ZAP's Viral Destruction by Cell Surface Receptors. Biomedical Research and Reviews. 2020; 3(2): 1-8.

[10] T. Ishida; Virucidal Activities of Zinc-Finger Antiviral Proteins and Zinc-Binding Domains for Virus Entry, DNA/RNA Replication and Spread. Edelweiss Journal of Biomedical Research and Review. 2020; Volume 2 Issue 1 | PDF 109 | Pages 1-5.

[11] M. Cassandri, A. Smirnov, F. Novelli,et al; Zinc-finger protein in health and disease. Cell Death Discovery. 2017; 3: 1-12.

[12] A. J. W. te Velthuis, S. H. E. van den Worm, A. C. Sims, et al; $\mathrm{Zn}^{2+}$ inhibits coronavirus and arterivirus RNA polymerase activity in vitro and zinc ionophores block the replication of these viruses in cell culture. PLOS Pathogen. 2010; 6, Issue 11: 1-10.

[13] N. Z. Gammoh and Lothar Rink (2017); Zinc in infection and inflammation, Nutrient, 9: 1-25.

[14] M. Maywald, Inga Wessels and L. Rink (2017); Zinc signals and immunity, J. Mol. Sci. 18:1-34.
[15] S. Belouzard, J. K. Millet, B. N. Licitra and G. R. Whittaker; Mechanisms of coronavirus cell entry mediated by the viral spike protein. Viruses. 2012; 4: 1011-1033.

[16] C. Wu, Y. Liu, Y. Yang, P. Zhang, et al; Analysis of therapeutic targets for SARS-CoV2 and discovery of potential drugs by computational methods. Acta Pharmaceutica Sinica B. 2020; 20: S2211-

[17] D. Wrapp, N. Wang, K. S. Corbett, et al; CryoEM structure of the 2019-nCoV spike in the prefusion conformation. Science. 2020; 367: 1260-1263.

[18] D. Schoeman and B. Fielding; Coronavirus envelope protein: current knowledge. Viology Journal. 2019; 69:1-22.

[19] A. Wu, P. Niu, L. Wang, H. Zhou, et al; Mutation, recombination and insertion in the evolution of 2019-nCoV. bioRxiv preprint. 2020; 29 February: 1-38.

[20] JOHNS HOPKINS BLOOMBERG SCHOOL of PUBLIC HEALTH; nCoV Genetics. 2020; Feb 3: 1-2.

[21] Changechuan Yin; Genotyping coronavirus SARS-CoV-2: methods and implications. ArXiv preprint. 2020; March 25: 1-12.

[22] Y. Gao, L. Yan, Y. Huang, F. Liu, et al; Structure of RNA-depndent RNA polymerase from 2019-nCoV, major antiviral drug target. BioRxiv preprint. 2020; 6: 1-24.

[23] Marc Lipsitch, Rebecca Kahn and Michael J. Mina; Antibody testing will enhance the power and accuracy of COVID-19-prevention trials. Nature Medicine. 2020;April 27:1-2. doi.org/ 10.1038/s41591-020-0887-3

[24] Mohammed S. Razzaque; COVID-19 pandemic: can maintaining optimal zinc balance enhance host resistance? Preprint 2020; 29 March:1-11.

[25] Amin Gasmi, Sadaf Noor, Torsak Tippairote, Maryam Dadar, et al; Individual risk management strategy and potential therapeutic options for the COVID-19 pandemic. Journal Pre-proof https://doi.org/10.1016/ j.clim.2020. 108409. 3-38.

[26] Chunyan Wanga, Wentao Lia, Dubravka Drabekb, et al;A human monoclonal antibody blocking SARS-CoV-2 infection. BioRxiv 2020; March 12; 1-24. https://doi.org/ 10.1101 /2020.03.11.987958

[27] Fang Li; Receptor Recognition Mechanisms of Coronaviruses: a Decade of Structural Studies. Journal of Virology. 2015; 89 No.4: 1954-1964.

[28] Anatoly V. SkalnY, Lothar Rink, OLGA P. Ajsuvakova, Michael Aschner, et al; Zinc and respiratory tract infections: Perspectives for COVID-19 Review). International Journal Of Molecular Medicine.2020;13April: 1-10.

[29] Emma Derbyshire, Joanne Delange;COVID-19: is there a role for immunonutrition, particularly 
Zinc( II ) Immune Virucidal Activities for 2019-nCoV Prevention and COVID-19 Respiratory Ailment and Pneumonia

in the over 65s? BMJ Nutritioh Prevention \& Health. 2020; 0: 1- 6. doi. org/ 10. 1136/ bmjnph- 2020- 000071.

[30] Manoj Kumar Gupta, Sarojamma Vemula, Ravindra Donde, Gayatri Gouda, Lambodar Behera \& Ramakrishna Vadde; In-silico approaches to detect inhibitors of the human severe acute respiratory syndrome coronavirus envelope protein ion channel. Journal of Biomolecular Structure and Dynamics. 2020; : 739-1102

[31] Markus Hoffmann, Hannah Kleine-Weber, Simon Schroeder, et al; SARS-CoV-2 Cell Entry Depends on ACE2 and TMPRSS2 and Is Blocked by a Clinically Proven Protease Inhibitor. Cell. 2020; 181: 271-280.

[32] A. J. W. te Velthuis, S. H. E. van den Worm, A. C. Sims, et al; $\mathrm{Zn}^{2+}$ inhibits coronavirus and arterivirus RNA polymerase activity in vitro and zinc ionophores block the replication of these viruses in cell culture. PLOS Pathogen. 2010; 6, Issue 11: 1-10.

[33] Karen Sargsyan, Ting Chen, Cédric Grauffel, and Carmay Lim; Identifying COVID-19 DrugSites Susceptible To Clinically Safe Zn-ejector Drugs Using Evolutionary /Physical Principles. 2020; Print:1-10.

[34] Derwand R and Scholz M; Does zinc supplementation enhance the clinical efficacy of chloroquine/hydroxychloroquine to win todays battle against COVID-19? Preprint doi:10.20944/preprints202004.0124.v1 2020; 8, April:1-10.

[35] Amir Noeparast, Gil Verschelden; Can Zinc correction in SARS-CoV-2 patients improve treatment outcomes? preprint, 2020; 7 April:114 doi:10.20944/preprints202004.0094.v1.

[36] J. L. Nieto-Torres, M. L. DeDiego, C. VerdiaBaguena, et al; Severe acute respiratotry syndrome coronavirus envelope ion channel activity poromotes virus fitness and pathogenesis. PLOS pathogens. 2014; 10, Issue 5: 1-19.

[37] Ying-Hui Jin1, Lin Cai, Zhen-Shun Cheng, Hong Cheng, Tong Deng, Yi-Pin Fan, et al; A rapid advice guideline for the diagnosis and treatment of 2019 novel coronavirus (2019$\mathrm{nCoV}$ ) infected pneumonia (standard version). Military Medical Research 2020; 7(4):1-23.

[38] Jianqing $\mathrm{Wu}$ and Ping Zha; Preventive, Mitigating and Treatment Strategies for Containing or Ending The COVID-19 Pandemic. Preprint for comments. 2020; Preprint for comment: 1-38.

[39] Jianqing Wu1, and Ping Zha; Treatment Strategies for Reducing Damages to Lungs In Patients with Coronavirus and Other Infections. Preprint 2020; 9 February: 1-31.

[40] Ghulam Shabbir Laghari, Zahid Hussain, Muhammad Taimur, Nasir Jamil; Therapeutic
Role of Zinc Supplementation in Children Hospitalized with Pneumonia. Open Access Original Article DOI: 10.7759/cureus. 4475. 1-6.

[41] UIS E. CUEVAS \& AI KOYANAGI; Zinc and infection: a review. Annals of Tropical Paediatrics. 2005; 25: 149-160.

[42] Meenakshi Kar, Naseem Ahmed Khan, Aleksha Panwar, Sachendra S. Bais, et al; Zinc Chelation Specifically Inhibits Early Stages of Dengue Virus Replication by Activation of NF$\kappa \mathrm{B}$ and Induction of Antiviral Response in Epithelial Cells. Frontiers in Immunity. 2019; 10: $1-15$.

[43] Cheng-Chung Lee, Chih-JungKuo, Tzu-Ping, et al; Structural Basis of Inhibition Specificities of 3C and 3C-like Proteases by Zinc-Coordinating and Peptidomimetic Compounds. Journal of Biological Chemistry. 2009; 284, No. 12: 7646-7655.

[44] The Rockefeller University; Virus entry and virus-host interactions. Laboratory of Virology and Infectious Disease. 2019; December 15: 1 page.

[45] W-Y. J. Lee, R. Menhua, C. Liang \& R. D. Sloan (2018); IFITM proteins inhibit HIV-1 protein synthesis. Scientific Reports. 8:1-15 pages.

[46] K. M. Crosse, E. Monson, M.R. Beard, K. J. Helbig (2018); Interferon-simulated genes as enhancers of antiviral innate immune signaling. Journal of Innate Immunity. 10: 85-93.

[47] M. J. Bick, J-W. N. Carroll, et al, (2003); Expression the zinc-finger antiviral protein inhibits Alphavirus replication. Journal of Virology. 77, No.21: 11555-11562.

[48] Q. Tang, X. Wang, G. Gao (2017); The short form of the zinc finger antiviral protein inhibits influenza A virus porotein expression and is antagonized by the virus-encoded NS1. Journal of Virology. 91,Issue 2: 1-14 pages

[49] F. Villalon-Letelier, A. G. Brooks, P. M. Saunders, et al (2017); Host cell restriction factors that limit influenza A infection. Viruses. 9(12):1-18pages.

[50] J. M. Schapiro, S. J. Libby, and F. Fang (2003); Inhibition of bacterial DNA replication by zinc mobilization during nitrosative stress. PNAS. 2003; 100, No.14: 8496-8501.

[51] Anne L. Sapiro, Emily C. Freund, Lucas Restrepo, Huan-Huan Qiao, et al; Zinc finger RNA binding protein Zn72D regulates ADARmediated RNA editing in neurons. BioRxiv reprint. doi: https://doi.org/10.1101/631986 2020; 8 May:1-43.

[52] A. Seybert, C. C. Posthuma, L. C. van Dinten, et al; A complex zinc finger controls the enzymatic activities of nidovirus helicases. Journal of Virology. 2005; 79, No.2: 696-704.

[53] L. M. John Law, B. S. Razooky, M. M. H. Li, et al (2019); ZAP's stress granule localization is 
Zinc( II ) Immune Virucidal Activities for 2019-nCoV Prevention and COVID-19 Respiratory Ailment and Pneumonia

correlated with its antiviral activity and induced by virus replication. PLOS Pathogens. 15(5): 1-22.

[54] Nathalie Sol-Foulon, Marion Sourisseau1, Franc, oise Porrot1, Maria-Isabel Thoulouze, et al (2007); ZAP-70 kinase regulate HIV cell-tocell spread and virological synapse formation. The EMBO Journal. 26: 516-526.

[55] W. Mothes, N. M. Sherer, J. Jin, P. Zhong (2010); Virus cell-to-cell transmission. Journal of Virology. 84, No.17: 8360-8368.

[56] X. Wang, F. Lv and G. Gao (2010); Mutagenesis analysis of the zinc-finger antiviral protein. Retrovirology. 7: 1-9 pages.

[57] G. Gao, X. Guo, S. P. Goff (2002); Inhibition of Retroviral RNA production by ZAP, a CCCH-type zinc finger protein. SCIENCE, 297: 1703-1706.

[58] Anne L. Sapiro, Emily C. Freund, Lucas Restrepo, Huan-Huan Qiao, et al; Zinc finger RNA binding protein $\mathrm{Zn72D}$ regulates ADARmediated RNA editing in neurons. BioRxiv reprint. doi: https://doi.org/10.1101/631986 2019; BioRxiv reprint:1-43.
[59] X. Luo, X. Wang, Y. Gao, et al; Molecular mechanism of RNA recognition by zinc-finger antiviral protein. Cell Reports. 2019; 30: 46-52.

[60] Xuemin Guo, Jing Ma, Jing Sun, and Guangxia Gao; The zinc-finger antiviral protein recruits the RNA processing exosome to degrade the target mRNA. PNAS. 2007; 104, no. 1: 151-156

[61] Ananda S. Prasad and Bin Bao; Molecular Mechanisms of Zinc as a Pro-Antioxidant Mediator: Clinical Therapeutic Implications. Antioxidants. 2019; 8:1-22.

[62] Olga A. Khomich, Sergey N. Kochetkov 1, Birke Bartosch and Alexander V. Ivanov; Redox Biology of Respiratory Viral Infections. Viruses. 2018; 10:1-27. doi:10.3390/v100 80392

[63] Aikaterini Nasi, Stephanie McArdle, Gustav Gaudernack, et al; Proteasome and reactive oxygen species dysfunction as risk factors for SARS-CoV-2 infection; consider Nacetylcystein as therapeutic intervention. FULLTEXT02. 2020;4-01:1-5.

Citation: Dr. Sci. Tsuneo Ishida, "Zinc( II) Immune Virucidal Activities for 2019-nCoV Prevention and COVID-19 Respiratory Ailment and Pneumonia”, International Journal of Research Studies in Medical and Health Sciences. 2020; 5(6): 21-33.

Copyright: (C) 2020 Dr. Sci. Tsuneo Ishida, This is an open-access article distributed under the terms of the Creative Commons Attribution License, which permits unrestricted use, distribution, and reproduction in any medium, provided the original author and source are credited. 